\author{
Nota científica \\ (Short Communication)
}

\title{
EFECTO DEL HONGO TRICHODERMA HARZIANUM SOBRE LAS ABEJAS SIN AGUIJÓN SCAPTOTRIGONA MEXICANA, MELIPONA BEECHEII, Y LA ABEJA MELÍFERA APIS MELLIFERA (HYMENOPTERA: APIDAE)
}

Recibido: 15/09/2015; aceptado: 29/01/2016

Toledo-Hernandez, RA., Saavedra-Santos, CJ., Betanzos-Simón, JE. \& Sánchez-Guillén, D. 2016. Activity of Trichoderma harzianum upon the stingless bees Scaptotrigona mexicana, Melipona beecheii, and the honey bee Apis mellifera (Hymenoptera: Apidae). Acta Zoológica Mexicana (n.s.), 31(2): 113-115.

ABSTRACT. Toxicity of the Trichoderma harzianum-based fungicide Bioben ${ }^{\circledR}$ was evaluated in three species of bees and Anastrepha ludens, in the Soconusco region, Chiapas, Mexico, under laboratory conditions. Mortality in Scaptotrigona mexicana and Apis mellifera were slightly higher in the exposed groups than in control groups, contrary to Melipona beecheii and A. ludens.

La antracnosis es una enfermedad causada por el hongo Colletotrichum gloesporioides (Penz) Penz. y Sacc., (Glomerellales: Glomerellaceae), la cual afecta a diversos cultivos, incluyendo el mango. Aunque su control se basa en el uso de fungicidas sintéticos, hace poco tiempo se empezaron a usar la bacteria Bacillus subtilis (Ehrenberg) Cohn (Bacillales: Bacillaceae) y el hongo saprófito Trichoderma spp. (Hypocreales: Hypocreaceae) como agentes de control en la región del Soconusco, Chiapas (Daniel Sánchez, comunicación personal). Aunque se considera que los microorganismos antagónicos usados para el control de fitopatógenos no representan un riesgo para los insectos polinizadores, una serie de estudios sugieren que cocteles de agroquímicos supuestamente no tóxicos pueden convertirse en un problema ambiental (Iwasa et al. 2004). El cultivo de mango no es la excepción, ya que durante las aplicaciones de B. subtilis y Trichoderma spp., también se asperjan insecticidas macrólidos y/ o organofosforados para el control de trips. Mommaerts et al. (2008), evaluaron los efectos de biofungicidas comerciales a base de Trichoderma harzianum Rifai y $T$. polysporum Rifai en el abejorro Bombus terrestris L., encontrando una baja mortalidad por vía oral y cuticular en adultos y larvas. Desafortunadamente no hay estudios que determinen el efecto de T. harzianum sobre especies de abejas diferentes a los géneros Apis o Bombus. Por tal razón el objetivo de este trabajo fue evaluar la mortalidad que causa T. harzianum sobre las abejas Scaptotrigona mexicana Guérin-Meneville, Melipona beecheii Bennet y como estándar de comparación se evaluó a Apis mellifera L. y Anastrepha ludens Loew.

Las pruebas de patogenicidad se realizaron en El Colegio de la Frontera Sur, en la ciudad de Tapachula, Chiapas, México, durante los meses de abril a mayo de 2015. Previo al experimento se evaluó la viabilidad del hongo usando la metodología descrita por Wraight et al. (2007). Se expusieron de manera tópica abejas forrajeras adultas de $S$. mexicana, M. beecheii y de A. mellifera, obtenidas de colonias que no recibieron ningún tratamiento con cualquier sustancia química y que se encontraban con suficientes crías y reservas, además de una población adulta numerosa. También se evaluó a una cepa de la mosca mexicana de la fruta Anastrepha ludens Loew (Diptera: Tephritidae) criada en el laboratorio con fines comparativos. Las abejas y las moscas se expusieron a la concentración de $T$. harzianum sugerida por el proveedor para el control de antracnosis (Bioben, Agrícola Innovación, México: $\left.1.2 \times 10^{9} \mathrm{UFC} / \mathrm{L}\right)$. Como tratamiento control se asperjó una solución de Tween al 0.1\% (componente inerte). Antes de la inoculación los especímenes se colocaron en un refrigerador a $5{ }^{\circ} \mathrm{C}$ por cinco minutos para facilitar su manejo. La inoculación se realizó colocando los insectos en una arena de exposición, la cual fue asperjada con una torre microaspersora que aplicaba la formulación bajo un flujo constante de aire a $1.41 \mathrm{~kg} / \mathrm{cm}^{2}$ (Mascarin et al. 2013). Para el caso de S. mexicana, A. mellifera y A. ludens se hicieron ocho repeticiones por especie, tres de las cuales correspondieron al tratamiento control. En cada repetición se expusieron 30 abejas. Para el caso de $M$. beecheii se hizo el mismo número de repeticiones, pero cada repetición consistió en 12 abejas, debido a la baja densidad de obreras en las colonias de esta especie. Una vez inoculados los insectos se introdujeron en un recipiente de plástico transparente ( $25 \mathrm{~cm}$ altura, $12 \mathrm{~cm}$ diámetro) 
y se mantuvieron en laboratorio a $28 \pm 2{ }^{\circ} \mathrm{C}$, en completa oscuridad las 24 horas y se alimentaron con agua-miel. Se hizo un seguimiento de la mortalidad de los especímenes durante ocho días. El porcentaje de mortalidad se verificó cada 24 horas: un insecto se consideró muerto si en estado inmóvil no respondía a la agitación del recipiente. Para verificar sí la causa de la muerte había sido el hongo, se tomaron los insectos muertos y se depositaron en cámaras húmedas durante 96 horas, se tomaron muestras de las abejas "micosadas" para detectar su infección por el hongo y se llevaron a cabo pruebas para la identificación de T. harzianum (Barnett \& Hunter 1999). Los resultados del porcentaje de mortalidad obtenidos se analizaron por ANOVA de una vía de medidas repetidas por especie.

La viabilidad de la formulación de $T$. harzianum fue del $90.1 \%$, por lo que los resultados obtenidos pueden considerarse confiables. El efecto causado por T. harzianum fue muy similar entre el tratamiento control y el expuesto al hongo en las tres especies de abejas y en $A$. ludens (Fig. 1). Para M. beecheii $\left(F_{1,6}=7.17, P=0.04\right)$ y $A$. ludens $\left(F_{1,6}=9.54, P=0.02\right)$ la mortalidad no fue significativa a $\alpha=0.01$. Sin embargo, la mortalidad en las abejas expuestas fue significativamente mayor en $S$. mexicana $\left(F_{1,6}=19.17, P<0.01\right)$ y $A$. mellifera $\left(F_{1,6}=\right.$ $24.19, P<0.01)$. Tanto para $S$. mexicana como para $A$. mellifera la diferencia se debió a la mortalidad en el tratamiento expuesto que fue mayor en los dos primeros días de exposición (prueba de Tukey HSD, $P<0.05$ ). En las cuatro especies la mortalidad varió significativamente en el tiempo $\left(F_{1,6}=19.17, P<0.001\right)$ y fue menor a $40 \%$ en el grupo expuesto y control al terminar los 8 días de observaciones. Los datos obtenidos indicaron que Bioben ${ }^{\circledR}$ presentó poca actividad sobre $A$. ludens y las tres especies de abejas. En un trabajo previo, Mommaerts et al. (2009) reportaron baja mortalidad $(<15 \%)$ en $B$. terrestris después de ser expuesto al biofungicida Trianum-P (formulación a base de T. harzianum cepa T22). Aunque la alta mortalidad que se observó en los primeros días en $S$.
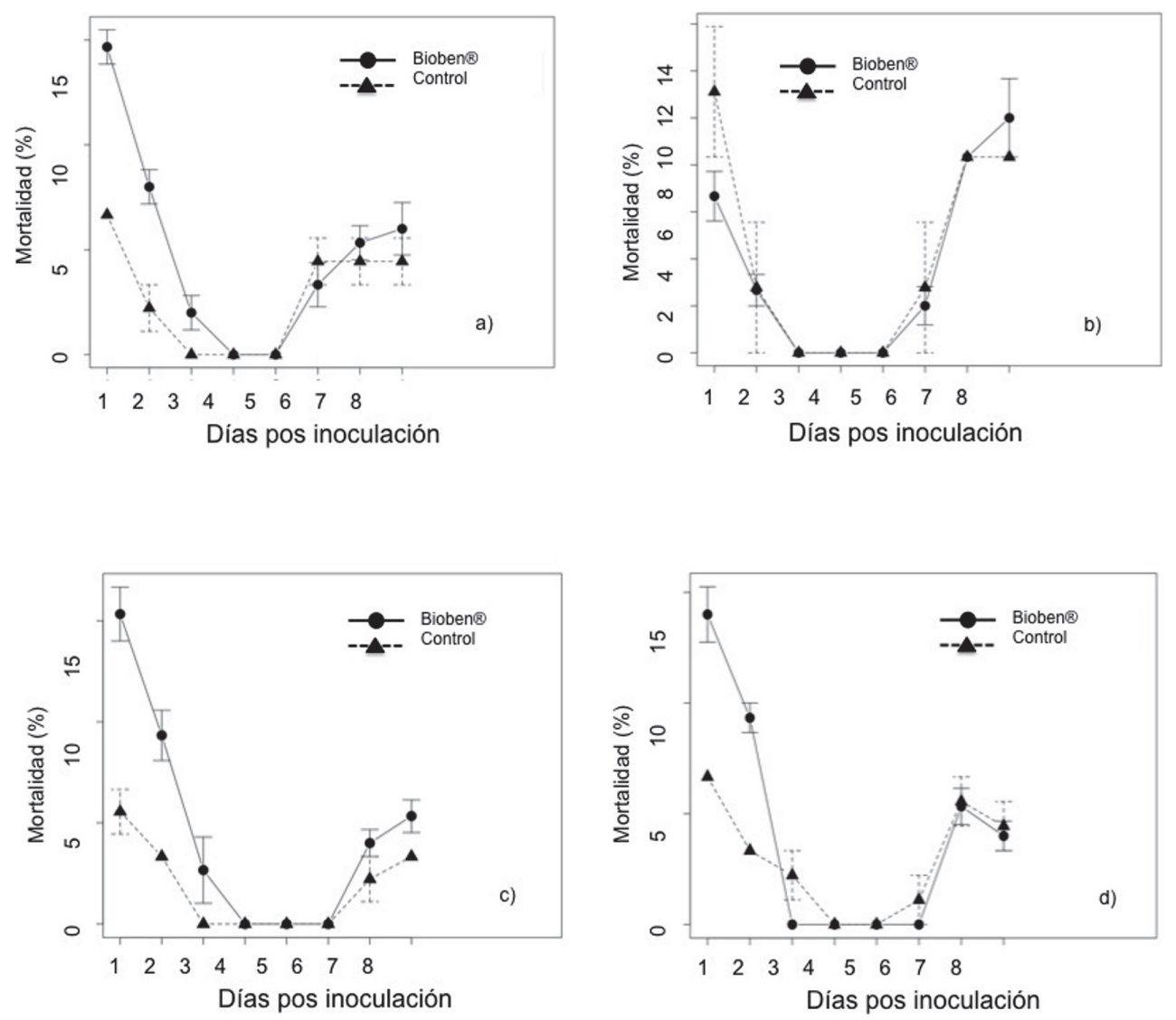

Figura 1. Mortalidad promedio ( \pm error estándar) de abejas y moscas expuestas al biofungicida Bioben ${ }^{\circledR}$ durante 8 días (a $=$ Scaptotrigona mexicana; $b=$ Melipona beecheii; $\mathrm{c}=$ Apis mellifera; $\mathrm{d}=$ Anastrepha ludens). 
mexicana y A. mellifera fue sugestivo de un efecto causado por el hongo, en los días subsecuentes no se observó el mismo patrón, lo que puede significar que algunas especies fueron susceptibles a los componentes inertes de la formulación. Este es un campo poco explorado pero que merece atención, ya que hay evidencia que indica que diferentes formulaciones provocan diferentes mortalidades (Mayes et al. 2003, Mohan \& Gujar 2001). Finalmente, los resultados del presente trabajo sugieren que la formulación comercial de T. harzianum (Bioben ${ }^{\circledR}$ ) no representa un riesgo para las abejas $S$. mexicana, $M$. beecheii y $A$. mellifera. Los siguientes pasos son: 1. Evaluar el efecto de este patógeno en combinación con otros plaguicidas usados para el control de plagas, a fin de descartar los posibles efectos sinérgicos que pudieran tener sobre las abejas (Iwasa et al. 2004) y 2. Determinar el efecto de los componentes de las formulaciones comerciales para evaluar su contribución a la toxicidad del compuesto.

AGRADECIMIENTOS. Este trabajo fue financiado por el proyecto "Validación y desarrollo de tecnología para el manejo integrado de moscas de la fruta en mango con enfoque en áreas grandes" de SAGARPA-CONACYT (número 163431).

\section{LITERATURA CITADA}

Barnett, H. L., \& Hunter, B. 1999. Ilustrated genera of imperfect fungi. Fourth edition. Aps press. Minneapolis, Minnesota, USA. $218 \mathrm{pp}$.
Iwasa, T., Motoyama, N., Ambrose, J. \& Ro, M. 2004. Mechanism for the differential toxicity of neonicotinoid insecticides in the honey bee, Apis mellifera. Crop Protection, 23: 371-378.

Mascarin, G. M., Quintela, E. D., Da Silva, E. G. \& Arthurs, S. P. 2013. Precision micro-spray tower for application of entomopathogens. BioAssay, 8: 1-4.

Mayes, M. A., Thompson, G. D., Husband, B. \& Miles, M. M. 2003. Spinosad toxicity to pollinators and associated risk. Reviews of Environmental Contamination and Toxicology, 179: 37-71.

Mohan, M. \& Gujar, G. T. 2001. Toxicity of Bacillus thuringiensis strains and commercial formulations to the diamondback moth, Plutella xylostella (L.). Crop Protection, 20: 311-316.

Mommaerts, V., Platteau, G., Boulet, J., Sterk, G. \& Smagghe, G. 2008. Trichoderma-based biological control agents are compatible with the pollinator Bombus terrestris: A laboratory study. Biological Control, 46: 463-466.

Mommaerts, V., Sterk, G., Hoffmann, L. \& Smagghe, G. 2009. A laboratory evaluation to determine the compatibility of microbiological control agents with the pollinator Bombus terrestris. Pest Management Science, 65: 949-955.

Wraight, S. P., Inglis, G. D. \& Goettel, M. S. 2007. Fungi. Pp 223248. In: Lacey, L.A. \& H.K. Kaya (Eds.). Field manual of techniques in invertebrate pathology. Springer, The Neetherlands.

\section{Ricardo Alberto TOLEDO-HERNÁNDEZ, CHRYSTIAN JARETH SAAVEDRA-SANTOS, JUAN EDUARDO BETANZOS-SIMÓN, DANIEL SÁNCHEZ-GUILLÉN*}

El Colegio de la Frontera Sur (ECOSUR), Departamento de Agricultura Sociedad y Ambiente, Carr. Antiguo Aeropuerto, Km 2.5, C.P. 30700 Tapachula, Chiapas, México.

*Autor para correspondencia: dsanchez@ecosur.mx 